International Journal of Modern Physics A

(C) World Scientific Publishing Company

\title{
On the thermal phase structure of QCD at vanishing chemical potentials
}

\author{
Sonia Kabana \\ SUBATECH, Ecole des Mines, 4 rue Alfred Kastler, 44307 Nantes, France. \\ Peter Minkowski \\ Albert Einstein Center for Fundamental Physics, Institute for Theoretical Physics, University of \\ Bern, Sidlerstrasse 5, CH-3012 Bern, Switzerland, and CERN, Theoretical Physics, CH-1211 \\ Geneva 23, Switzerland. \\ Received Day Month Year \\ Revised Day Month Year
}

\begin{abstract}
The hypothesis is investigated, that the thermal structure of QCD phases at and near zero chemical potentials is determined by long range coherence, inducing the gauge boson pair condensate, and its thermal extension, representing a fundamental order parameter. A consistent model for thermal behaviour including interactions is derived in which the condensate does not produce any latent heat as it vanishes at the critical temperature inducing a second order phase transition with respect to energy density neglecting eventual numerically small critical exponents. Localization and delocalization of color fields are thus separated by a unique critical temperature.
\end{abstract}

Keywords: QCD; quark-gluon plasma; quantum phase transitions.

PACS numbers: 12.38.-t,12.38.Mh,05.30.Rt

\section{Introduction}

The existence and nature of the QCD phase transition is theoretically accessible to a universal description underlying a thermal system with all its general and specific restrictions. At the moment key theoretical questions concerning the phase structure of thermal systems in QCD remain. Lattice QCD calculations lead to very clear predictions, establishing the lack of any phase transition at zero chemical potentials 12 . The validity of this method and its results is not uncontroversial, for example see Refs. 34 .

There is a longstanding experimental effort to measure characteristic signatures of the phase transition in collisions of heavy ions at several facilities at AGS at BNL, SPS at CERN, RHIC at BNL, LHC at CERN and in the future FAIR at GSI. The present state of these studies yields significant results, well interpretable as a strongly interacting partonic matter formed in the earlier stages of the heavy ion collisions 516 . These results suggest the existence of a phase transition in the 
course of the collision. Assuming the correctness of this conclusion, the detailed phase structure and order(s) of assocated transition(s) could not be determined conclusively so far .

The experimentally measured reactions cover a large range of collision energies and chemical potentials in thermal analyses. In particular, with increasing energies the baryochemical potential in hadron-hadron or heavy ion collisions decreases. For a comparison reducing these systems to a common baryochemical potential we refer to 78 .

In this paper we propose to give an "outline in principle" of the thermal phases of QCD at vanishing chemical potentials and their relation to the gauge boson pair condensate of QCD. We proceed to show that the two phases, separated by the critical temperature $T_{c r}$ at $\mu=0$ can indeed be understood as a dynamical consequence of uniquely the bosonic pair condensate at nonzero quark masses, in contrast to supersymmetric models with no spontaneous breaking of supersymmetry.

The two phases are represented approximatively by a collection of noninteracting hadrons for $0<T<T_{c r}$ and a collection of 8 gauge bosons and 4 flavours of quarks and antiquarks (u,d,s,c \& anti) for $T>T_{c r}$, in which interactions are modeled. We use here initially two collections of hadron resonances, denoted Ntype $=65$ and Ntype $=26$ in the following. The Ntype $=65$ collection, on which most of the subsequent calculations of thermodynamic state functions rely, is an extension of the smaller Ntype $=26$ one. In the former $($ Ntype $=65)$ the $4 S U 3_{f l}$ meson nonets with $J^{P C_{n}}=0^{++}, 1^{++}, 2^{++} ; 1^{+-}$in the lowest mass p-wawe $q \bar{q}^{\prime}$ configuration are added, as well as the lowest mass open charm and anticharm s-wave pseudoscalar and vector mesons with composition $q \bar{c}$ and $c \bar{q}$ and baryons and antibaryons containing one charmed quark and their antiparticles together with overall s-wave composition $q q^{\prime} c$ and $\overline{q q} \bar{c}$ with $q, q^{\prime}=u, d, s$ are added, together with 3 representative color gauge boson binaries (glueballs) with $J^{P C}=0^{++}, 0^{-+}, 2^{++}$ and two hybrid nonets of composition $q g \bar{q}^{\prime}$ with $J^{P C_{n}}=1^{-+}$.

The adopted approximation allows to illustrate the thermal phase properties in principle.

\section{Energy momentum density tensor and gauge boson condensate}

We begin with the local, symmetric and conserved energy momentum density tensor $\vartheta_{\mu \nu}$ existing and renormalized as a basic consequence of QCD . The above properties imply exact Poincaré invariance.

$$
\left\{\vartheta_{\mu \nu}=\vartheta_{\nu \mu}\right\}(x) ; \partial^{\nu} \vartheta_{\mu \nu}=0
$$

The central defining quantities, of the existence and order of the phase transition, are pressure, energy density and entropy density for zero chemical potentials. The 
local operator form of the trace anomaly in QCD reads:

$$
\begin{aligned}
& \vartheta_{\mu}^{\mu}=\sum_{f} m_{f} S_{\dot{f f}}(x)+\delta_{0}(x) \\
& \delta_{0}=\left(-2 \beta(g) / g^{3}\right)\left[-\frac{1}{4}(:) F_{\mu \nu}^{A}(x) F^{\mu \nu A}(x)(:)\right]_{s . d .} \\
& =-2 b_{0}\left[\frac{1}{4}(:) F_{\mu \nu}^{A}(x) F^{\mu \nu A}(x)(:)\right]
\end{aligned}
$$

In Eq. 2 the subscript s.d. stands for "renormalization scale dependent", while $b_{0}=$ $9 /\left(16 \pi^{2}\right)$. All quantities are defined to be renormalized and renormalization group invariant, except those with the subscript s.d. $\beta(g)$ denotes the (Callan-Symanzik-) rescaling function, where $g$ is the coupling constant. $\frac{1}{4}(:) F_{\mu \nu}^{A} F^{\mu \nu A}(:)$ is the local scalar density - to be called local gauge boson bilinear - composed bilinearly of field strength tensors $F_{\mu \nu}^{A}$ - the latter including multiplicatively the gauge coupling constant in their definition, relative to the perturbative field strength normalization. $A$ denotes the color component within the adjoint representation of $S U(3)_{c} \cdot(:)(:)$ stands for a suitable normal ordering. $m_{f}$ denotes the mass of quark flavour f : $S_{\dot{f} f}$ denotes the local scalar density of antiquark $\dot{f}$ and quark $f$ flavors :

$S_{\dot{f} f}(x)=(:) \bar{q}_{\dot{f} \dot{c} s}(x) q_{f c}^{s}(x)(:)$, where s denotes the spin.

The focus is to consider vacuum expected values of the local operators in Eq. 2 which by translation invariance are independent of the position $x$, suppressed in the following

$$
\begin{aligned}
& \eta^{\mu \nu}\left\langle\Omega\left|\vartheta_{\mu \nu}(x)\right| \Omega\right\rangle=-2 b_{0}\left\langle\Omega\left|\frac{1}{4}(:) F_{\mu \nu}^{A} F^{\mu \nu A}(x)(:)\right| \Omega\right\rangle \\
& +m_{f}\left\langle\Omega\left|S_{\dot{f f}}(x)\right| \Omega\right\rangle
\end{aligned}
$$

$\left\langle\Omega\left|(:) \frac{1}{4} F_{\mu \nu}^{A} F^{\mu \nu A}(:)\right| \Omega\right\rangle=\mathcal{B}^{2}$ shall be called the gauge boson pair condensate , abbreviated by $\mathcal{B}^{2}$.

$\left\langle\Omega\left|S_{\dot{f} f}\right| \Omega\right\rangle \neq 0$ induces spontaneous chiral symmetry breaking and is generally called quark condensate.

In connection with normal ordering ambiguities it is important to admit in the precise form of the energy momentum tensor a nontrivial vacuum expected value , which as a consequence of exact Poincaré invariance must be of the form

$$
\begin{aligned}
& \left\langle\Omega\left|\vartheta_{\mu \nu}(x)\right| \Omega\right\rangle=-\eta_{\mu \nu} p_{v a c} \\
& \left\{\eta_{\mu \nu}=\operatorname{diag}(1,-1,-1,-1) ; p_{v a c}=-\rho_{v a c}\right\} \\
& \text { independent of } x \rightarrow \\
& \Delta \vartheta_{\mu \nu}(x)=\vartheta_{\mu \nu}(x)-\left\langle\Omega\left|\vartheta_{\mu \nu}(x)\right| \Omega\right\rangle|\Omega\rangle\langle\Omega| \\
& \text { with } \partial^{\nu} \Delta \vartheta_{\mu \nu}(x)=0 ;\left\langle\Omega\left|\Delta \vartheta_{\mu \nu}(x)\right| \Omega\right\rangle=0
\end{aligned}
$$

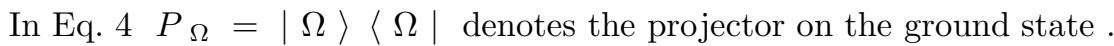

From the two local, conserved tensors in Eq. 4 only $\Delta \vartheta_{\mu \nu}(x)$ with vanishing vacuum expected value is acceptable as representing the conserved 4 momentum operators and their densities yielding the integral form

$$
\widehat{P}_{\mu}=\int{ }_{t} d^{3} x \Delta \vartheta_{\mu 0}(t, \vec{x})
$$


We use here throughout strictly thermal , 'extension in phase space' associated potentials, depending in subtle ways on vacuum condensates. To these potentials no vacuum associated spontaneous parameters like $p_{v a c}=-\rho_{v a c}$, defined in Eq. 4. contribute in a direct way, dominating in the limit $T \rightarrow 0 ; \mu_{\alpha} \equiv 0$.

From Eqs. 3 and 4 we obtain the relation and estimates 'pour fixer les idées'

$$
\begin{aligned}
p_{\text {vac }} & =\frac{9}{32 \pi^{2}} \mathcal{B}^{2}+\frac{1}{4} \Lambda=\left\{\begin{array}{l}
0.00302 \mathrm{GeV}^{4} \\
0.00658 \mathrm{GeV}^{4}
\end{array}\right. \\
\mathcal{B}^{2} & =\left\{\begin{array}{l}
0.125 \mathrm{GeV}^{4}[9] \\
0.250 \mathrm{GeV}^{4} 10
\end{array}\right. \\
\Lambda & =-\sum_{f} m_{f}\left\langle\Omega\left|S_{\dot{f f}}\right| \Omega\right\rangle \\
& \sim f_{\pi}^{2}\left(\frac{1}{2} m_{\pi}^{2}+m_{K}^{2}\right)=0.00217 \mathrm{GeV}^{4}
\end{aligned}
$$

\section{Construction of a thermal model including interactions}

We follow the strategy layed out in Refs. $\frac{78}{18}$ taking into account the modifications described above, distinguishing two eventual phases

1) the hadronic (h)-phase, with color localized within stable hadrons and selected hadron resonances corresponding to the two collections Ntype $=65 \subset 26$. Thermal potentials of the (h)-phase are approximated by those of free hadrons, neglecting decay widths, as described in Refs. 78 .

2) the quark-antiquark-gauge boson (qg)-phase, wherein thermal potentials are related but not equal to those of free quarks and antiquarks, restricted to the flavors $\mathrm{u}, \mathrm{d}, \mathrm{s}$ and $\mathrm{c}$, and 8 gauge bosons pertaining to the gauge group $S U 3_{c}$. Next we describe the modeling of interactions in the (qg)-phase, which deviates from noninteracting constituents assumed in Refs. $\frac{78}{18}$.

We introduce for later use for the (qg)-phase, the Gibbs density $g_{q g}^{(0)}$ and energy density $\varrho_{e}^{(0)} q g$ pertaining to noninteracting tricolored quarks, antiquarks with flavors

$\mathrm{u}, \mathrm{d}, \mathrm{s}, \mathrm{c}$ and eightfold colored gauge bosons

$$
\begin{aligned}
& g_{q g}^{(0)}(T)=\sum_{\alpha_{q g}} w_{\alpha_{q g}}\left(1 /\left(2 \pi^{2}\right)\right) \int_{m_{\alpha_{q g}}}^{\infty} l E p d E \\
& w_{\alpha_{q g}}=\left(2 \operatorname{spin}_{\alpha_{q g}}+1\right)\left\{\begin{array}{l}
3 \text { for } q, \bar{q} \\
8 \text { for } g
\end{array}=\left\{\begin{array}{c}
6 \text { for } q, \bar{q} \\
16 \text { for } g
\end{array}\right.\right. \\
& \beta \equiv 1 / T ; l=\mp \log [1 \mp \exp (-\beta E)] \\
& \varrho_{e q g}^{(0)}(T)=-(d / d \beta) g_{q g}^{(0)}(T)=T^{2}(d / d T) g_{q g}^{(0)}(T)
\end{aligned}
$$

In Eq. 7 the index $\alpha_{q g}$ runs over the different constituents of the (qg) phase, while $w_{\alpha_{q g}}$ denotes the multiplicity beyond momentum phase space associated with the constituent $\alpha_{q g}$. The sign ( $\mp$ ) in the expression for $l$ is - for bosons and + for fermions . 
We choose the following masses for quark flavors $\mathrm{u}, \mathrm{d}, \mathrm{s}, \mathrm{c}$

$$
\begin{aligned}
{\left[m_{u}\right.} & =0.00525, m_{d}=0.00875 \\
m_{s} & \left.=0.175, m_{c}=1.27\right] \mathrm{GeV}
\end{aligned}
$$

The absolute masses of the $\mathrm{u}, \mathrm{d}, \mathrm{s}$ light flavors as well as their ratios $m_{u}: m_{d}$ : $m_{s}=3: 5: 100,11|12| 13$, used here play no decisive role in the present derivations, within generous ranges of $\pm 20 \%$.

The inclusion of the charmed quark serves the purpose to check whether it has any significant influence on the thermal parameters in the region of $T_{c r} \sim 0.2 \mathrm{GeV}$, wich turns out to be in the few percent range .

We proceed to modify the free quark antiquark gauge boson (qg-) parametrization of the Gibbs potential and the energy density, which for $\mu_{\alpha}=0$ must obey the exact relation

$$
\begin{aligned}
& g_{q g}=g_{q g}\left(T \equiv \beta^{-1}\right) ;-(d / d \beta) g_{q g}(T)=\varrho_{e q g}(T) \\
& \text { and } g_{q g} \leftrightarrow g_{h}, \varrho_{e q} \leftrightarrow \varrho_{e} h
\end{aligned}
$$

The Gibbs- and energy-densities in the hadron phase are constructed from the expressions analogous to the ones given in Eq. 7, where the index $\alpha_{q g} \rightarrow \alpha_{h}$ runs over a suitable choice of hadrons and hadron resonances as defined in Refs. $\frac{17 / 8}{6}$ with real masses and neglecting interactions among these states .

The ensuing parametrization of interactions is understood as representing the phase structure in principle and not in numerical detail. The modeling of interactions in the qg-phase is performed setting two parameters $k, \Delta g$, independent of temperature, as approximately parametrizing the interaction in the deconfined phase in a limited region of $T \geq T_{c r}$

$$
\begin{aligned}
& \varrho_{e q g}(T ; k)=k \varrho_{e}^{(0)}{ }_{q g}(T) \\
& g_{q g}(T ; k, \Delta g)=k g_{q g}^{(0)}(T)-\Delta g
\end{aligned}
$$

The parameter $0<k<1$ is taking into account the reduction of Gibbs density or pressure relative to the noninteracting (Stefan-Boltzmann) limit, noted in perturbative QCD calculations of thermal parameters for $T \simeq T_{c r}$ of interest here 14, while the second parameter $\Delta g$ arises as integration constant from the differential equation ( Eq. 9), which is clearly satisfied for arbitrary values of $(k, \Delta g)$.

We proceed in two steps to map out the structure of the phase transition

I : the condition determining $T_{c r} \leftrightarrow k$

The equality of the energy densities - in the hadron phase $\varrho_{e}$ had for $T \leq$ $T_{c r} \quad$ as outlined in Refs. 78 and in the qg-phase as defined in Eq. 10 $\varrho_{e ~ q g}$ for $T \geq T_{c r}$ determine the critical temperature $\varrho_{e}$ had $(T)=\varrho_{e q g}(T ; k) \leftrightarrow T=T_{c r}(k)$

The matching ( Eq. 11) is further restricted to yield the value

$$
T_{\text {cr }} \sim 0.2 \mathrm{GeV} \leftrightarrow k \sim 0.365
$$

in accordance with the estimate of one of us 16 . 
II : the condition avoiding singular behaviour of pressure gradient

This condition implies

$$
g_{h}\left(T_{c r}\right)=g_{q g}\left(T_{c r} ; k, \Delta g\right) \leftrightarrow \Delta g=\Delta g\left(T_{c r}(k)\right)
$$

and determines $\Delta g$

$$
\Delta g \sim 0.0062 \mathrm{GeV}^{3}=0.775 \mathrm{fm}^{-3}
$$

\section{Results and discussion}

We begin with a disussion with a comparison of the two colletions of hadron resonances Ntype $=65$ and Ntype $=26$ whence thermal state functions are extrapolates beyond the temperature range $130 \mathrm{MeV} \leq T \leq 170 \mathrm{MeV}$ obtained in studies of chemical equilibrium of hadron yield ratios.

It is expected that approaching a phase transition will require interactions on the hadronic side as well as on the quark-gluon side. The mere extension of the collection of hadron resonances also beyond Ntype $=65$ here, without introduction of interactions among the hadron resonances is unrealistic. If there is no phase transition at all, this does not change the mentioned expectation.

This is illustrated in Figs. 1 and 2 , in which the two noninteracting collections of hadron resonances, Ntype $=65$ and 26 , are compared with respect to the quantities dscale $=\left(\varrho_{e}-3 p\right) / T^{4}$ and pressure $/ T^{4}$ respectively .

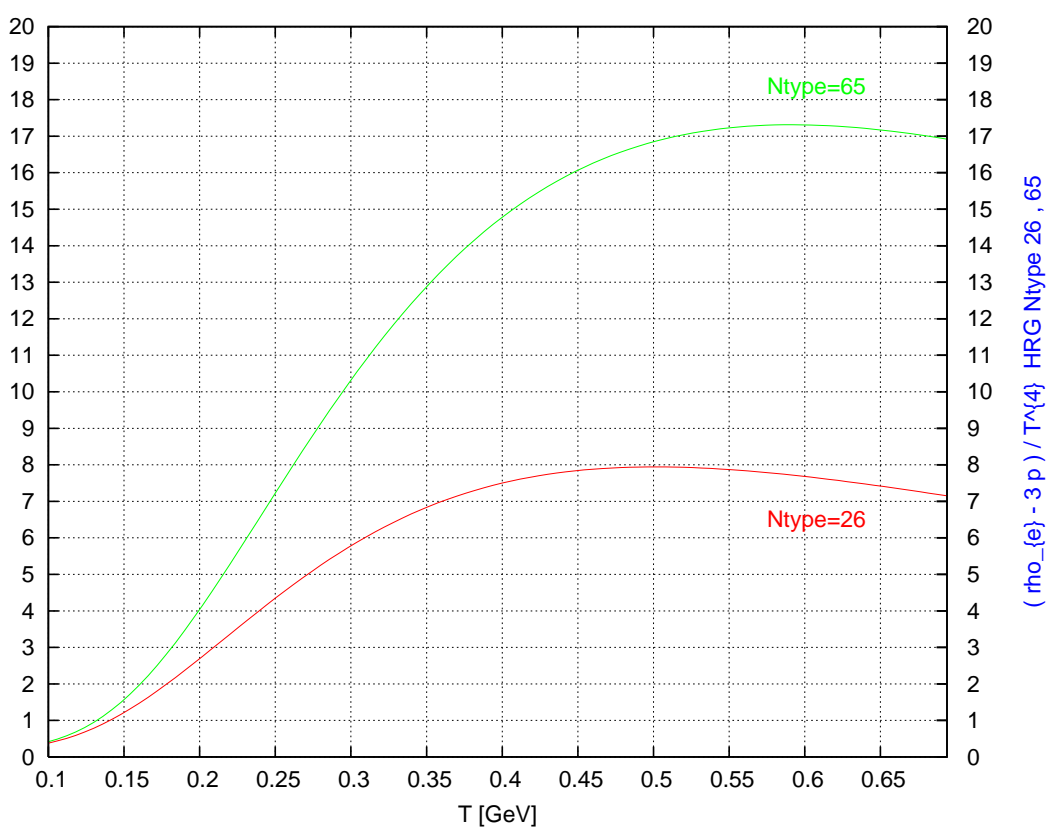

Fig. 1. The thermal quantity representing the trace anomaly-dscale $(T)=\left(\varrho_{e}-3 p\right) / T^{4}$ is shown for $0.1 \mathrm{GeV} \leq T \leq 0.7 \mathrm{GeV}$. The comparison of HRG collections Ntype=65 and 26 shows the sensitive temperature regions beyond temperatures where chemical freeze out takes place $-T \sim 150-170 \mathrm{MeV}$ for $\mathrm{Pb}-\mathrm{Pb}$ collisions at SPS and $\mathrm{Au}-\mathrm{Au}$ collisions at RHIC. 


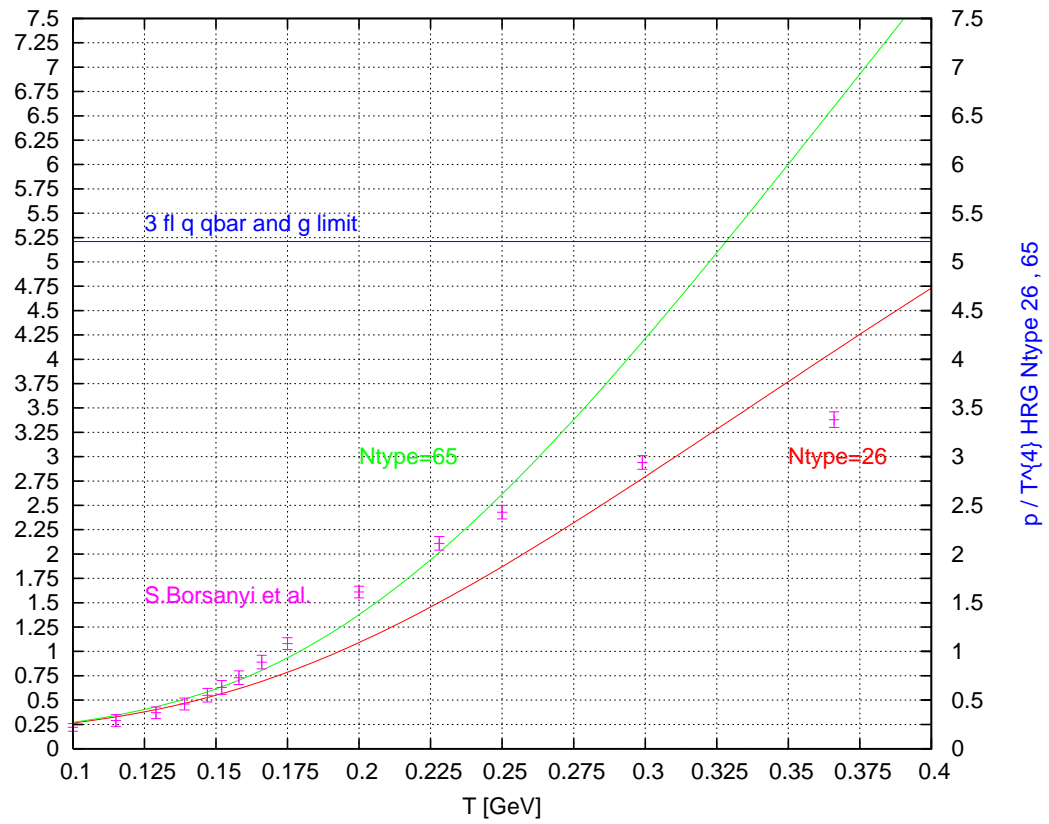

Fig. 2. Pressure / $T^{4}$ versus $\mathrm{T}$ in the range $0.1 \mathrm{GeV} \leq T \leq 0.4 \mathrm{GeV}$, for the two collections Ntype $=65$ and 26 is compared with the results of lattice QCD by S. Borsanyi et al. 28 .

As a consequence of the fair agreement of the pressure $/ T^{4}$ as obtained from the noninteracting hadron resonance collection Ntype $=65$ with the lattice simulation results of S. Borsanyi et al. $\frac{128}{}$, as shown in Fig. 2 , we choose this collection in all subsequent calculations representing the hadron phase for $T \leq T_{c r} \simeq 200 \mathrm{MeV}$. We mention the fact, that collections of hadron resonances used in recent analyses of especially ratios od hadron yields as observed in Au-Au collisions at RHIC include all resonances reported by the PDG 15 up to a mass of $2 \mathrm{GeV}$ or $2.5 \mathrm{GeV}$, which are larger than the one defined Ntype $=65$ here. In Ref. 1 the agreement up to $T \sim 170 \mathrm{MeV}$ of all thermal state functions with the HRG quantities of these large collections is very satisfactory .

The restriction to noninteracting hadron resonances nevertheless has a limited validity particularly at temperatures above chemical freeze out as $T \sim 200 \mathrm{MeV}$. 
The result of steps I and II above are illustrated in Figs. 3 - 7 below . For a comparison with thermodynamic analyses in lattice QCD we refer to Refs. 17, 18 and 1 . The existence of two crossing points, one each for energy density and pressure, coincident in temperature at acceptable values of all parameters near $T_{c r} \simeq 0.2 \mathrm{GeV}$ is nontrivial, shown in Fig. 3 below

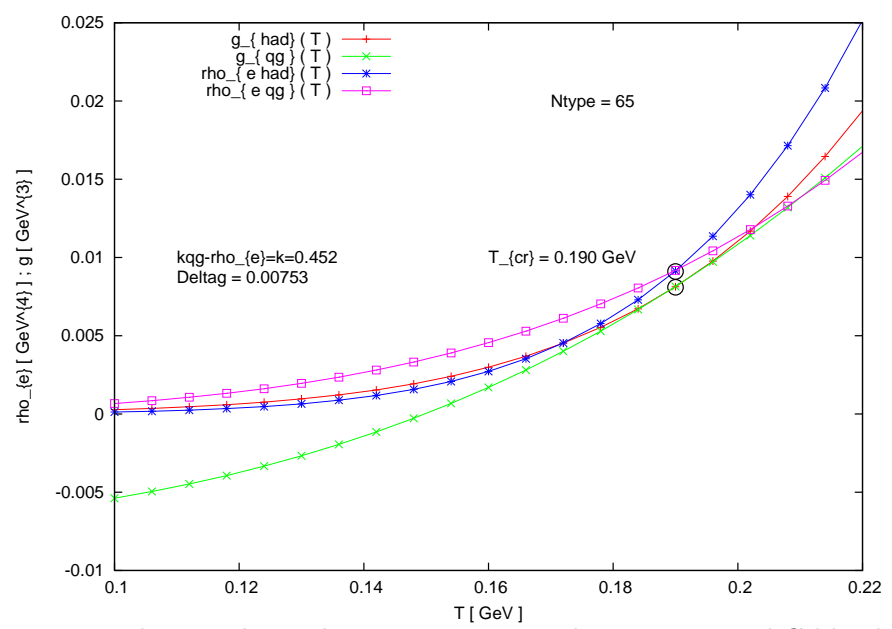

Fig. 3. The two thermal quantities energy density $\varrho_{e}$ and Gibbs density $g=p / T$ in units of $\mathrm{GeV}^{4}$ and $\mathrm{GeV}^{4}$ respectively are decomposed into the hadronic (had) (valid for $T \leq T_{c r}$ ) and quark-gluon (qg) parts ( valid for $T \geq T_{c r}$ ) each : $\varrho_{\text {e had }}$ : blue curve; $\varrho_{\text {e } q g}$ : purple curve and $g_{\text {had }}:$ red curve ; $g_{q g}:$ green curve. The two small circles mark the two equalities $\varrho_{e \text { had }}=\varrho_{e q g}$ and $g_{h a d}=g_{q g}$ coincident in temperature $T=T_{c}\left(\equiv T_{c r}\right)$.

In Figs. $4-6$ the modifications of $\varrho_{e q g} / T^{4}, p_{q g} / T^{4}$ and dscale = $\left(\varrho_{e q g}-3 p_{q g}\right) / T^{4}$ introduce a subleading critical exponent $\nu ; \nu=0.975$ used here throughout, in the vicinity of $T=T_{c r}$ allowing the free quark-gluon limits to be reached for $T \rightarrow \infty$. The modified quantities are defined as

$$
\begin{aligned}
& \left\{\begin{array}{l}
\varrho_{e}^{\nu} q g \\
p_{q g}^{\nu}
\end{array}\right\}=\left\{\begin{array}{l}
f \bmod \left(\nu, T / T_{c}\right) \varrho_{e q g}+f \bmod 1\left(\nu, T / T_{c}\right) p_{q g} \\
\operatorname{fmod}\left(\nu, T / T_{c}\right) p_{q g}
\end{array}\right\} \\
& f \bmod \left(\nu, T / T_{c r}\right)=1+\left|1-T_{c r} / T\right|^{2 \nu}(1 / k-1) \\
& \operatorname{fmod} 1\left(\nu, T / T_{c}\right)=T(d / d T) \operatorname{fmod}\left(\nu, T / T_{c}\right)
\end{aligned}
$$

Assuming that all thermodynamic potentials are analytic functions of $T$ in a sufficiently large neighbourhood of $T_{c r}$, with the transition arising from appropriate conditional choices below and above $T_{c r}$, as is the case for the parametrizations adopted here, the orders of the so deduced phase transition ( for $\mu_{\alpha}=0$ ) become 


\begin{tabular}{ccl} 
order & with respect to & quantity \\
\hline 2. & $\leftrightarrow$ & energy density \\
3. & $\leftrightarrow$ & pressure \\
1. & $\leftrightarrow$ & sound velocity
\end{tabular}

We note the behaviour of the square velocity of sound. Steps I and II imply that the sound velocity (square) exhibits a 1 . order transition at $T=T_{c r}$.

Let the squares of the sound velocity in the hadron phase ( qg-phase), always for vanishing chemical potentials, be denoted respectively

$$
v_{s \text { had }(q g)}^{2}(T)=\frac{\dot{p}_{\text {had }(q g)}}{\dot{\varrho}_{e \text { had }(q g)}} ; \cdot=d / d T
$$

It follows that the sound velocity (square) jumps from a lower value in the hadron phase to a higher value in the qg-phase .

For the qg-phase we have the relations

$$
\begin{aligned}
& p_{q g}=T\left(k g_{q g}^{(0)}-\Delta g\right), T g_{q g}^{(0)}=p_{g q}^{(0)}(T) \\
& \dot{p}_{q g}^{(0)}=\varrho_{s}^{(0)}(T): \quad \begin{array}{l}
\text { entropy density } \\
\text { for noninteracting } \\
\text { qg-phase }
\end{array} \\
& \longrightarrow v_{q g}^{2}=\frac{k \dot{p}_{q g}^{(0)}-\Delta g}{k \dot{\varrho}_{e}^{(0)}}=\left(v_{q g}^{2}\right)^{(0)}-\frac{\Delta g}{k \dot{\varrho}_{e}^{(0)} q g}
\end{aligned}
$$

All quantities with superscript (0) refer to the noninteracting qg-phase . The introduction of interactions leads to a reduction of the qg-sound velocity square proportional to $\Delta g$. This is however a small correction for all $T>T_{c r}$ as shown in Fig. 4, also illustrating the 1. order transition of $v_{s}^{2}$. In the qg-phase the sound velocity square approaches the limiting value $\frac{1}{3}$ and is just after the transition already quite near this value. The behaviour of $v_{s}^{2}$ is shown in Fig. 7 . 


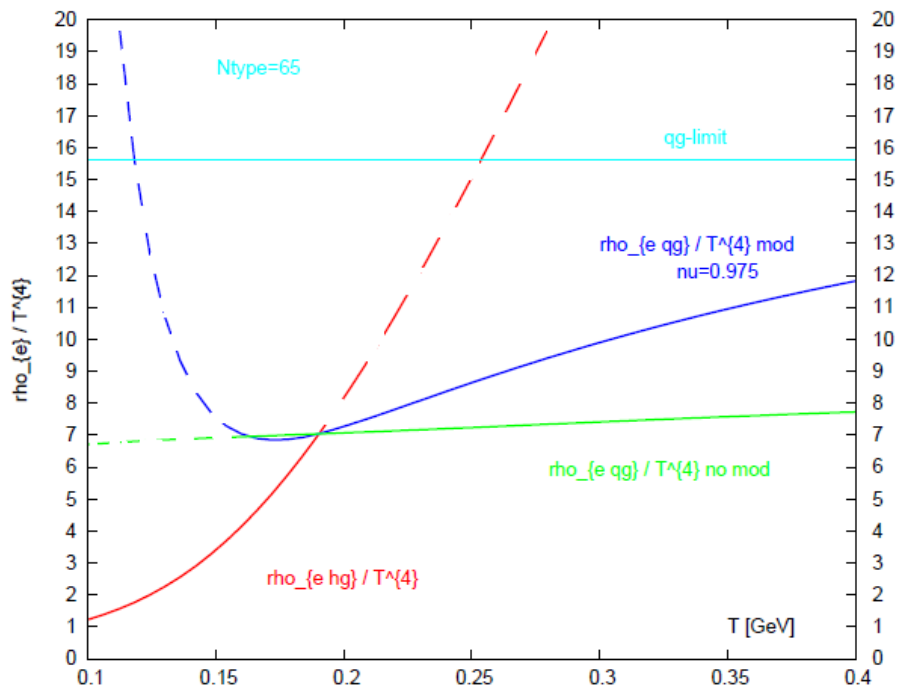

Fig. 4. The ( dominantly ) second order nature is displayed of the transition - unmodified and modified on the quark-gluon-side by subleading critical exponents - of the quantity $\varrho_{e}(T) / T^{4}$, piecewise described by $\varrho_{e} h g(-65) / T^{4}$ for $T \leq T_{c r}$ from the HRG side and $\rho_{\text {e qg no mod }(\bmod )} / T^{4}$ for $T \geq T_{c r}$ from the quark-gluon side as defined in Eq. 15 The three curves are represented with dashed lines outside their range of validity.

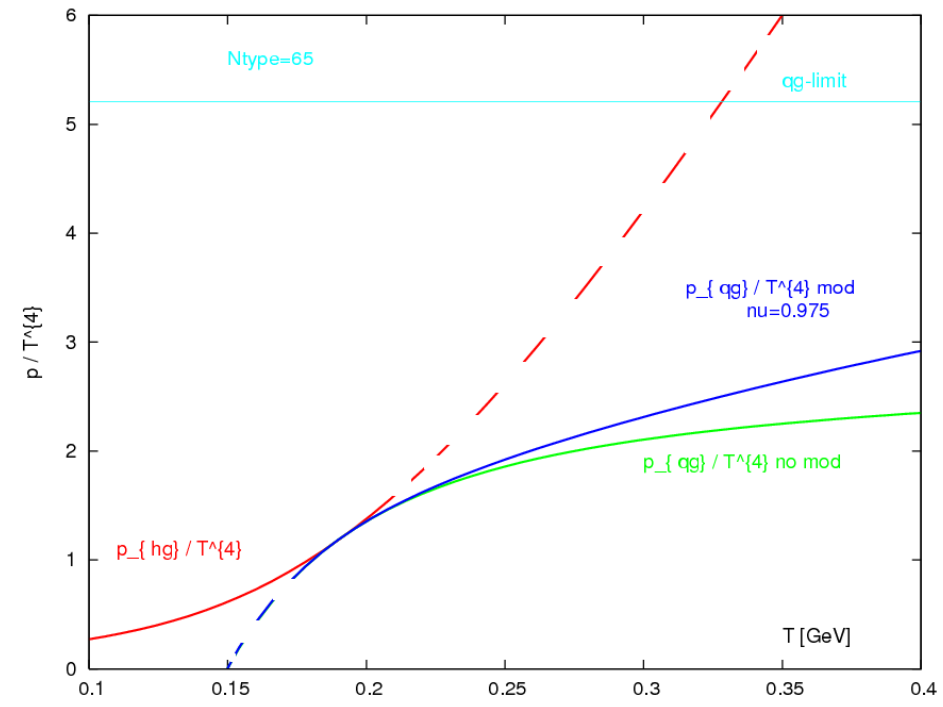

Fig. 5. The ( dominantly ) third order nature is displayed of the transition under the same conditions as underlying Fig. 4 for the quantity $p / T^{4}$, piecewise described by $p_{\text {e }} h g(-65) / T^{4}$ for $T \leq T_{c r}$ from the HRG side and $p_{q g \text { no } \bmod (\bmod )} / T^{4}$ for $T \geq T_{c r}$ from the quarkgluon side as defined in Eq. 15. The three curves are represented with dashed lines outside their range of validity. 


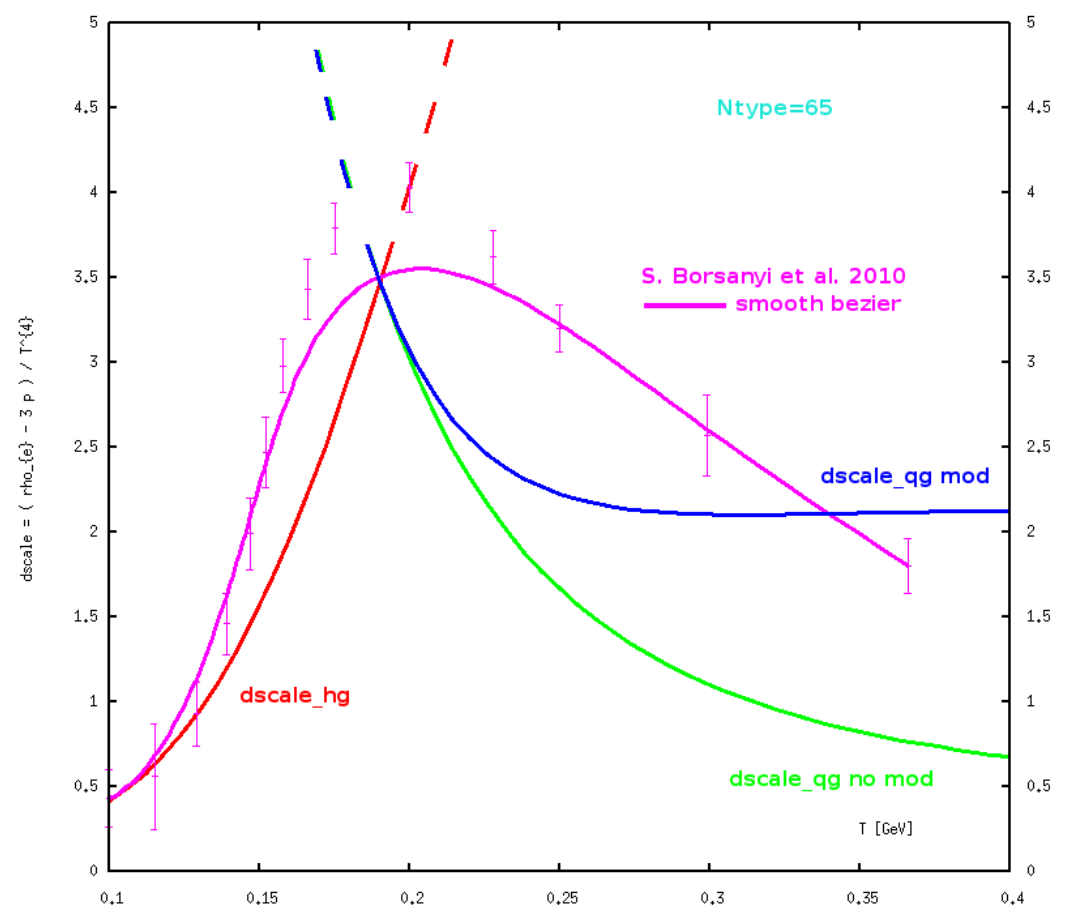

Fig. 6. The ( dominantly ) second order nature of the transition is displayed with respect to the thermal quantity - dscale - forming the shape of an 'indian tent'. The same quantity as obtained in Ref. 28 from lattice simulation of QCD under the same thermal conditions is also plotted for comparison. The quantity dscale ${ }_{q g \text { mod }}^{\nu}$ is obtained from Eq. 15, here with $\nu=0.975$, the same as in Figs. $4-5$. The three curves forming the 'indian tent' are represented with dashed lines outside their range of validity. 


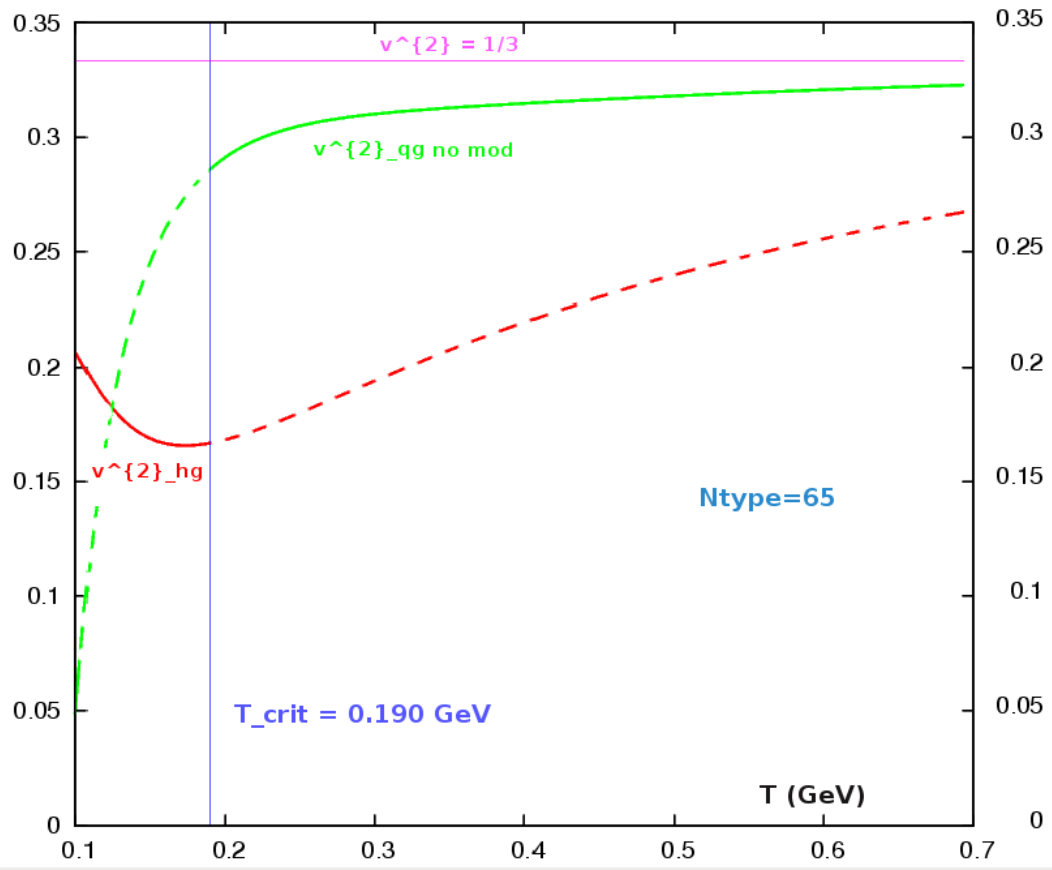

Fig. 7. The ( dominantly ) first order nature is displayed of the transition with respect to the square of the velocity of sound, under the same conditions as for Figs. 4 - 6, piecewise described by the quantities $v_{h g}^{2} ;(h g \equiv h)$ for $T \leq T_{c r}$ from the HRG side and $v_{q g \text { no mod }}^{2}$ for $T \geq T_{c r}$ from the quark-gluon side. Only the unmodified setting is used for $v_{g q}^{2}$. The two curves for $v_{h g}^{2}, v_{g q}^{2}$ are represented with dashed lines outside their range of validity. 
The derivations concerning the phase structure of QCD at vanishing chemical potentials, laid out in the present discussion, follow within the hypothesis, that the gauge boson pair condensate is at the origin of stable embeddings of quark flavors - light and heavy - into the ( long range ) dynamics controled primarily by gauge boson self-interactions. By the same hypothesis - this embedding depends in a minimal way on quark masses, including their chiral as well as anti-chiral limits : $m_{\chi_{1}}, \cdots m_{\chi_{M}} \rightarrow 0 ; m_{h_{1}}, \cdots m_{h_{N}} \rightarrow \infty$.

Extensive lattice simulations of the thermodynamic state in particular at zero chemical potential have been performed in recent years. With realistic quark masses for the three light flavours $\mathrm{u}, \mathrm{d}, \mathrm{s}$ no phase transition but a cross over region is found. For a review we refer to Ref. [13].

For pure gauge theories with gauge group $S U N_{c}, N_{c}>2$ a 1st order phase transition and for $N_{c}=2$ a second order transition is found [14,15].

The latter phase transition agrees with the results pertaining to the 3-dimensional Ising model.

\section{The argument for - at least - a second order phase transition with respect to energy density}

In this subsection a short outline is given of how spontaneous parameters at $T=0$ and $T>0$ are obtained in general renormalizable local field theories.

We define first in physical space-time, the effective action $\Gamma$ for the composite classical field $\varphi_{c l} \leftrightarrow \varphi$ associated with the local gauge boson bilinear, introduced in Eq. 2

$$
\begin{aligned}
& \exp i W=\left\langle\Omega\left|\exp \left(i \int d^{4} x(\mathcal{L}+\mathcal{J} \varphi)(x)\right)\right| \Omega\right\rangle_{c} \\
& \varphi(x)=\frac{1}{4}(:) F_{\mu \nu}^{A}(x) F^{\mu \nu} A(x)(:) ; \mathcal{J}(x): \text { source }
\end{aligned}
$$

in the presence of a classical local source $\mathcal{J} \cdot{ }_{c}$ in Eq. 19] denotes the connected part of the generating functionsal $W$. The effective action $\Gamma\left(\varphi_{c}\right)$ is obtained from the generating functional $W$ through functional Legendre transform

$$
\begin{aligned}
& \varphi_{c l}(x)=\delta / \delta \mathcal{J}(x) W(\mathcal{J}) \rightarrow \\
& \Gamma\left(\varphi_{c l}\right)=\int d^{4} x\left(\varphi_{c l}(x) \mathcal{J}(x)\right)-W ; \varphi_{c l}=\varphi_{c l}(\mathcal{J}) \\
& \delta / \delta \varphi_{c l}(x) \Gamma=\mathcal{J}(x) ;\left.\varphi_{c l}(x)\right|_{\mathcal{J} \rightarrow 0}=\mathcal{B}^{2},(\text { Eqs. 316) } \\
& \Gamma\left(\varphi_{c l}\right)=\int d^{4} x v\left(\varphi_{c l}\right)
\end{aligned}
$$

The (nonlocal) density functional $v$ defined in Eq. 20 determines the spontaneous ground state expected value of $\varphi_{c l}$ in the limit of vanishing sources as absolute minimum.

It is essential to safeguard appropriate boundary and/or integrability conditions for the sources $\mathcal{J}(x)$ which translate into corresponding conditions for the Legendre 
transforms $\varphi_{c l}(x)$, e.g.

$$
\lim _{x \rightarrow \infty} \mathcal{J}(x)=0 \leftrightarrow \int d^{4} y|\mathcal{J}|^{2}(y)<\infty
$$

as described in Ref. 20. The functionals $W, \Gamma$ in Eqs. 19, 20 can be extended to Euclidean space. The absolute thermodynamic limit at zero temperature is reached from the boundary conditions in Eq. 21 for spontaneous vacuum parameters allowing sources and classical fields to approach also constant values 21, whereas thermal environment at finite temperature $T$ corresponds to a finite Euclidean time extension

$$
\Delta t_{E}=\beta=1 / T
$$

In the finite temperature thermal limit new boundary conditions at the bounding times $t_{E}=0, \beta$ have to be set, periodic for gauge invariant bosonic sources and classical fields, as considered above relative to the local operator $\varphi(x)$ defined in Eq. 19. Hence we face the two thermodynamic limits, treated here for just the associated quantities

$$
\begin{aligned}
& \frac{1}{4}(:) F_{\mu \nu}^{A}(x) F^{\mu \nu A}(x)(:)=\varphi(x) \rightarrow\left(\varphi_{c l}(x), \mathcal{J}(x)\right) \\
& \mathcal{J}(x)=\left(1 / g^{2}\right)-\left(1 / g^{2}(x)\right)=-\Delta \frac{1}{g^{2}}(x)
\end{aligned}
$$

The external source $\mathcal{J}(x)$ represents a space time dependent coupling constant, before thermodynamic limits are taken.

Absolute thermodynamic ( $T \equiv 0)$ - and thermal finite temperature ( $T$ finite ) limits yield the relations

$$
\begin{aligned}
& \mathcal{J}(x) \quad \underset{ }{\rightarrow} \begin{array}{l}
J \in\left[-J_{*}, J_{*}\right] \rightarrow 0 \\
v\left(\varphi_{c l}(x)\right)^{T} \\
v\left(\varphi_{c l}, T\right)
\end{array} \mid T:\left\{\begin{array}{l}
\equiv 0 \\
\text { finite }
\end{array}\right. \\
& \partial_{\varphi_{c l}} v\left(\varphi_{c l}, T\right)=J \rightarrow 0 \rightarrow \varphi_{c l}=\varphi_{c l}(T)
\end{aligned}
$$

Performing the sequence of limits and letting the (constant) values of the source $J$ vary in a suitable interval $\left[-J_{*}, J_{*}\right]$ before relaxing it to zero, as shown in Eq. 24 , defines the hysteresis line of the spontaneous parameter $\varphi_{c l}(J \rightarrow 0, T)$. The latter emerges as thermal average for $T$ finite and as vacuum expected value for $T \equiv 0$ (Eqs. 19, 211).

In the $T$ finite case we have the choice to include chemical potentials, one each for conserved quark flavor neutral currents, which we set to zero here.

The phase transition underlying the present discussion is associated - by hypothesis - with the vanishinging in a singular way at finite critical temperature, $T=T_{c r}>0$ of the quantity $\varphi_{c l}(T)$ defined in Eq. 24

$$
\varphi_{c l}(T) \rightarrow 0 \text { for } T \rightarrow T_{c r}
$$


Classical configurations leading to this behaviour inherit a 'Watt-less' nature from beeing particular to the ground state of QCD $22 \mid 23$ and thus should not generate a step-like behaviour of thermal energy density. This is borne out in the subtraction of the ground state projection of the associated energy momentum density tensor $\vartheta_{\mu \nu}$ as shown in Eqs. 415.

This however does not imply that the first derivative of energy density changes by a finite amount through the transition, i.e. its corresponding genuinely second order nature. The singularity could well be characterized by critical exponents without relation to a definite step in a given derivative - of thermal energy density.

The mechanism of gauge boson pair condensation discussed here is a new analysis taking its roots in material presented in Refs. 22,16 and 718 in chronological order. It is centered on the thermal average of the field strength bilinear local density operator defined in Eq. 2

$$
\frac{1}{4}(:) F_{\mu \nu}^{A}(x) F^{\mu \nu A}(x)(:)
$$

The nonzero vacuum expected value ( Eq. 3 ) signals a connection between the localization of color and the structure of Bogoliubov transformations as appropriate for gauge fields at long range.

For QCD, actual calculations of effective potentials at large and intermediary range are not accessible to perturbation theory. Hence analytic control is lacking at present.

\section{Conclusions}

Our modeling of strong interactions in the qg-phase, in the vicinity but above the critical temperature $T_{c r} \sim 0.2 \mathrm{Mev}$, as described between Eqs. 10 and 18, is supported by observations of strong coupling in central Au-Au collisions at $200 \mathrm{GeV}$ at RHIC 5[27. The reduction of pressure and energy density relative to noninteracting (anti-) quarks and gauge bosons then allows for the phase transition to be (essentially) of second order with respect to energy density, as demonstrated in the related thermal quantities shown in Figs. 2 and 3. The square of the velocity of sound reveals most directly the order of the transition through its discontinuity as shown in Fig. 4.

The upcoming lower c.m. energy scan at RHIC, new results expected from LHC concerning hadronic physics and more extended studies in lattice QCD promise to clarify its phase structure, eventually accompanied by theoretical insights.

\section{Acknowledgements}

It is a pleasure to thank the TH-division of CERN for its hospitality, and Anne Perrin as well as Markus Moser, representing the group of computing coordinators at the ITP in Bern, for their logistics support. Topical discussions with Martin Lüscher and Uwe-Jens Wiese are gratefully acknowledged. 


\section{References}

1. S. Borsanyi, Z. Fodor, C. Hoelbling, S. D. Katz, S. Krieg, C. Ratti, K. K. Szabo (Wuppertal-Budapest Collaboration), 'Is there still any Tc mystery in lattice QCD? Results with physical masses in the continuum limit III', 20. May 2010, arXiv:1005.3508 [hep-lat] .

2. The MILC Collaboration (A. Bazavov et al.), 'Results from the MILC collaboration's $\mathrm{SU}(3)$ chiral perturbation theory analysis', Oct 2009. 7pp., to appear in the proceedings of 27th International Symposium on Lattice Field Theory (Lattice 2009), Beijing, China, 25-31 July 2009, PoS LAT2009 (2009) 079, arXiv:0910.3618 [hep-lat] .

3. H. Neuberger, 'The "hard" problem of strong of interactions', 20. May 2010, arXiv:1005.3509 hep-lat] .

4. G. Rossi, M. Testa, 'A 0-dimensional counter-example to rooting?', Physics Letters B (2010), 21. May 2010, arXiv:1005.3672 [hep-lat] .

5. STAR coll., Nucl. Phys. A 757 (2005) 102,

Brahms coll., Nucl. Phys. A 757 (2005) 1,

Phenix coll., Nucl. Phys. A 757 (2005) 184,

Phobos coll., Nucl. Phys. A 757 (2005) 28.

6. M.C. Abreu et al., 'Anomalous J/ $\Psi$ suppression in $\mathrm{Pb}-\mathrm{Pb}$ interactions at $158 \mathrm{GeV} / \mathrm{c}$ per nucleon", Physics Letters B 410 (1997) 337-343,

F. Sikler et al., 'Hadron production in nuclear collisions from the NA49 experiment at $158 \mathrm{GeV} / \mathrm{c}$ A', Nuclear Physics A 661 (1999) 45c-54c,

G. Agakichiev et al., 'Low-mass e+ e- pair production in $158 \mathrm{~A} \mathrm{GeV} \mathrm{Pb-Au} \mathrm{collisions}$ at the CERN SPS, its dependence on multiplicity and transverse momentum', Physics Letters B 422 (1998) 405-412,

E. Andersen et al., 'Strangeness enhancement at mid-rapidity in $\mathrm{Pb}-\mathrm{Pb}$ collisions at 158 A GeV/c', Physics Letters B 449 (1999) 401-406,

R. Albrecht et al., 'Limits on the production of direct photons in $200 \mathrm{~A} \mathrm{GeV}{ }^{32} \mathrm{~S}+\mathrm{Au}$ collisions', Physical Review Letters 76 (1996) 3506-3509,

I.G. Bearden et al., 'Collective expansion in high-energy heavy ion collisions', Physical Review Letters 78 (1997) 2080-2083,

G. Ambrosini et al., 'Impact parameter dependence of $\mathrm{K}, \mathrm{p}, \overline{\mathrm{p}}$, d and $\overline{\mathrm{d}}$ production in fixed target $\mathrm{Pb}+\mathrm{Pb}$ collisions at $158 \mathrm{GeV}$ per nucleon', New Journal of Physics 1 (1999) 22.1-22.23

7. S. Kabana and P. Minkowski, 'Mapping out the QCD phase transition in multiparticle production', BUTP-2000-26, BUHE-00-09, Oct 2000, 53pp., New J.Phys.3 (2001) 4, hep-ph/0010247.

8. S. Kabana, 'The Strange border of the QCD phases', Eur.Phys.J.C21 (2001) 545-555.

9. M. Shifman, A. Vainshtein and V. Zakharov, 'QCD and Resonance Physics. Sum Rules', Nucl. Phys. B147 (1979) 385 .

10. S. Narison, 'Masses, decays and mixings of gluonia in QCD,' PM-96-37, Dec 1996. 32pp., Nucl. Phys. B509 (1998) 312 , hep-ph/9612457.

11. P. Minkowski, A. Zepeda, 'Hadron Masses And Current Algebra Quark Masses', Print79-0413 (BERN), May 1979, 35pp., Nucl.Phys.B164 (1980) 25 .

12. C. A. Dominguez, 'Determination of light quark masses in QCD', Comments: Plenary talk at the Conference in honour of Murray Gell-Mann's 80th birthday, Nanyang Technical University, Singapore, February 2010. To be published in the proceedings (Mod. Phys. Lett. A). This talk is based on work done in collaboration with N. F. Nasrallah, R. H. Röntsch, and K. Schilcher, 21. May 2010, arXiv:1005.3724 [hep-ph] .

13. S. Narison (CNRS-IN2P3, Montpellier), 'Gluon condensates and c, b quark masses 
from quarkonia sum rules', arXiv:1004.5333v3 [hep-ph] 6 May 2010 .

14. A. Ipp, K. Kajantie, A. Rebhan and A. Vuorinen, 'Unified description of deconfined QCD equation of state', J. Phys. G: Nucl. Part. Phys. 34 (2007) 631 and references cited therein .

15. Particle Data group, K. Nakamura et al. (Particle Data Group), J. Phys. G 37 (2010) 075021 .

16. P. Minkowski, 'Color Superconductivity In Qcd And The Associated Phase Transition', Bern university preprint BUTP-88-22, Sep 1988. 52pp., Czech.Hadron Symp.1988:279 .

17. M. Cheng, N.H. Christ, P. Hegde, F. Karsch, Min Li, M.F. Lin, R.D. Mawhinney, D. Renfrew, P. Vranas, 'The finite temperature QCD using 2+1 flavors of domain wall fermions at $N_{t}=8$ ', arXiv:0911.3450 [hep-lat].

18. A. Bazavov et al., CU-TP-1189, Mar 2009, (Received Mar 2009), 31pp., 'Equation of state and QCD transition at finite temperature', Phys.Rev.D80 (2009) 014504, arXiv:0903.4379 [hep-lat].

19. J. Noronha - Hostler, J. Noronha and C. Greiner, 'Transport Coefficients of Hadronic Matter near $T_{c}$ ', Phys.Rev.Lett.103 (2009) 172302, arXiv:0811.1571v4 [nucl-th].

20. M. Leibundgut and P. Minkowski, 'On the spontaneous identity of chiral and supersymmetry breaking in pure super Yang-Mills theories', BUTP-97-22, Aug 1997, 30pp., Nucl.Phys.B531 (1998) 95, hep-th/9708061

21. J. Zinn-Justin, 'Quantum field theory and critical phenomena', The International Series of Monographs on Physics, Vol. 113, Oxford University Press, Oxford 2002.

22. P. Minkowski, 'On the ground-state expectation value of the field strength bilinear in gauge theories and constant classical fields', Nucl.Phys.B177 (1981) 203.

23. H. Leutwyler, ' Constant gauge fields and their quantum fluctuations', Nucl. Phys. B179 (1981) 129 .

24. U. M. Heller, 'Recent progress in finite temperature lattice QCD', Plenary talk at 24th International Symposium on Lattice Field Theory (Lattice 2006), Tucson, Arizona, 23-28 Jul 2006, PoS LAT2006 (2006) 011, hep-lat/0610114

25. L. G. Yaffe and B. Svetitsky , 'First Order Phase Transition in the SU(3) Gauge Theory at Finite Temperature', CALT-68-913, (Received May 1982). 8pp., Phys.Rev.D26 (1982) 963.

26. B. Lucini, M. Teper and U. Wenger, 'Properties of the deconfining phase transition in SU(N) gauge theories', DESY-05-021, Feb 2005. 50pp., JHEP 0502 (2005) 033.

27. E. V. Shuryak, Nucl. Phys. A 750, 64 (2005), arXiv:hep-ph/0405066.

28. Szabolcs Borsanyi, (Wuppertal U.), Gergely Endrodi, (Eotvos U.), Zoltan Fodor, (Wuppertal U. \& Eotvos U.), Antal Jakovac, (Wuppertal U.), Sandor D. Katz, (Eotvos U.), Stefan Krieg, (Wuppertal U. \& MIT, LNS), Claudia Ratti, Kalman K. Szabo, (Wuppertal U.), 'The QCD equation of state with dynamical quarks', WUB-10-12, Jul 2010. 31pp., arXiv:1007.2580 [hep-lat]. 